\title{
Ectopic cervical thymi and no thymic involution until midlife in naked mole rats
}

\author{
Stephan Emmrich $^{1}$ (1) | Frances Tolibzoda Zakusilo ${ }^{1}$ Alexandre Trapp ${ }^{1}$ | Xuming Zhou ${ }^{2}$ | \\ Quanwei Zhang $^{3}$ | Ellen M. Irving ${ }^{1}$ | Michael G. Drage ${ }^{4}$ | Zhengdong Zhang ${ }^{3}$ |

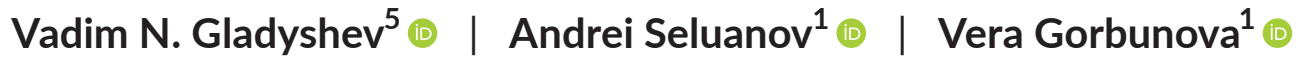

${ }^{1}$ Department of Biology, University of Rochester, Rochester, NY, USA

${ }^{2}$ CAS Key Laboratory of Animal Ecology and Conservation Biology, Institute of

Zoology, Chinese Academy of Sciences, Beijing, China

${ }^{3}$ Department of Genetics, Albert Einstein College of Medicine, New York City, NY, USA

${ }^{4}$ Pathology and Laboratory Medicine, University of Rochester Medical Center, Rochester, NY, USA

${ }^{5}$ Division of Genetics, Department of Medicine, Brigham and Women's Hospital, Harvard Medical School, Boston, MA, USA

\section{Correspondence}

Stephan Emmrich, University of Rochester, 436 Hutchison Hall, River Campus, Rochester NY 14627-0211, USA.

Email: stephan-emmrich@gmx.net

Andrei Seluanov, University of Rochester, 432 Hutchison Hall, River Campus, Rochester, NY 14627-0211, USA.

Email: andrei.seluanov@rochester.edu

Vera Gorbunova, University of Rochester, 434 Hutchison Hall, River Campus,

Rochester NY 14627-0211, USA.

Email: vera.gorbunova@rochester.edu

Funding information

National Institute on Aging

\begin{abstract}
Immunosenescence is a hallmark of aging and manifests as increased susceptibility to infection, autoimmunity, and cancer in the elderly. One component of immunosenescence is thymic involution, age-associated shrinkage of the thymus, observed in all vertebrates studied to date. The naked mole rat (Heterocephalus glaber) has become an attractive animal model in aging research due to its extreme longevity and resistance to disease. Here, we show that naked mole rats display no thymic involution up to 11 years of age. Furthermore, we found large ectopic cervical thymi in addition to the canonical thoracic thymus, both being identical in their cell composition. The developmental landscape in naked mole rat thymi revealed overt differences from the murine T-cell compartment, most notably a decrease of $\mathrm{CD} 4^{+} / \mathrm{CD} 8^{+}$double-positive cells and lower abundance of cytotoxic effector $\mathrm{T}$ cells. Our observations suggest that naked mole rats display a delayed immunosenescence. Therapeutic interventions aimed at reversing thymic aging remain limited, underscoring the importance of understanding the cellular and molecular mechanisms behind a sustained immune function in the naked mole rat.
\end{abstract}

\section{KEYWORDS}

aging, CD4-CD8 Ratio, immunosenescence, lymphopoiesis, naked mole rat, thymus, T-

lymphocytes

\section{1 | INTRODUCTION}

Age-related loss of cellularity and weight of the thymus, termed thymic involution, occurs in all mammals (Shanley et al., 2009). Thymic involution contributes to immunosenescence, leading to increased incidence of cancers, autoimmunity, and opportunistic infections in the elderly (Dixit, 2012; Lynch et al., 2009). Thymic involution remains an evolutionary mystery since it occurs in most vertebrates despite its negative effects. In vertebrates, aging of the thymus gland precedes age-associated phenotypic changes in other organs,

This is an open access article under the terms of the Creative Commons Attribution License, which permits use, distribution and reproduction in any medium, provided the original work is properly cited.

(c) 2021 The Authors. Aging Cell published by Anatomical Society and John Wiley \& Sons Ltd. 
with thymic function gradually decreasing from the first year of human life (Shanley et al., 2009). The process of human thymic involution is incompletely understood, in part due to a lack of animal models showing delayed immunosenescence (Tong et al., 2020).

Naked mole rats (NMR) display exceptional longevity, with a maximum lifespan of 32 years (Buffenstein, 2008; Buffenstein \& Jarvis, 2002), without increased mortality due to aging (Ruby et al., 2018). In comparison, a similarly sized house mouse has a maximum lifespan of 4 years (de Magalhaes et al., 2005; Turturro et al., 1999). NMRs display negligible senescence (Buffenstein, 2008), characterized by very slow changes in physiological parameters with age, and the lack of an age-related increase in mortality rate (Finch, 1990). Accordingly, NMRs do not exhibit age-related changes in basal metabolism, body composition, or bone mineral density (Buffenstein \& Ruby, 2021; O'Connor et al., 2002). NMRs show a very low incidence of cancer (Buffenstein, 2008; Delaney et al., 2013, 2016). NMRs produce abundant high molecular weight hyaluronic acid (HMW-HA) responsible for their resistance to solid tumors (Tian et al., 2013, 2015) and feature a serum metabolome resembling calorically restricted mice(Lewis et al., 2018; Puppione et al., 2021). Thus, NMRs present a unique model to study the mechanisms of healthy longevity (Edrey et al., 2011; Gorbunova et al., 2014). However, the information about the immune system of NMRs is limited. Very recently, whole spleen single-cell RNA-sequencing revealed the absence of canonical natural killer cells (NKC) in NMRs (Hilton et al., 2019). It was also reported that blind mole rats, an unrelated clade of subterranean rodents that convergently evolved extreme longevity, sustain sustained T-cell repertoire diversity in old age (Spalax spp.; Izraelson et al., 2018). At present, there are, however, no data regarding the T-cell compartment in NMRs.

Here, we set out to characterize the thymus and T-Lymphopoiesis in long-lived NMRs in comparison with short-lived mice. Our results revealed unexpected presence of additional cervical thymi in the NMR and the absence of thymic involution until 11 years of age, which is the oldest age of animals in our research colony.

\section{RESULTS}

Thymic involution and decline of T-cell function predisposes to opportunistic diseases and presents a major risk factor in the elderly (Dixit, 2012; Torroba \& Zapata, 2003). Since NMRs are characterized by negligible senescence, we set out to examine whether they display thymic involution. To enable the analysis of the NMR hematopoietic cells, we previously developed a flow cytometry (FACS) staining with cross-reactive monoclonal antibodies (moAbs) and functionally characterized purified hematopoietic stem and progenitor (HSPC) fractions (Emmrich et al., 2019). Through CITE-Seq, we showed that naked mole-rat T cells (TC) express CD3, LAT, and LCK are immunophenotypically Thy $1.1^{\text {int }} /$ CD $34^{-} /$CD $11 b^{-}$(Figure S1a,b).

Thymus glands extracted from the thoracic cavity of young NMRs and mice both featured similar sized lymphocytes and largely absent myeloid cell fractions (Figure S2a). When we extracted lymph nodes (LN) from 43 naked mole-rat necks, we noted two types of cervical nodes (Figure 1a): the first type corresponding to $L N$ and the second resembling a thymus, which we termed cervical thymi. Thoracic and cervical thymi contained a $\mathrm{CD} 34^{+}$fraction while LNs did not, and shared histological features such as many medullary sinuses, no germinal centers and more basophilic cytoplasms than LNs (Figure 1b,c). Conceivably, both thymi types stain positive for cytokeratin as seen for mouse and human thymi, while LNs are cytokeratin ${ }^{-}$with a much lower TC:BC ratio (Figure 1d, Figure S2b). Embryonic thymus development proceeds through detachment of the primordium, which contains thymus and parathyroid anlagen, from the 3rd pharyngeal pouches, followed by separation of the anlagen and migration of thymic lobes into the chest cavity (Gordon \& Manley, 2011). We compared the macroscopic anatomy with mice and found undersized thoracic thymus lobes in NMR neonates (Figure S2c). To our surprise, the cervical thymus was clearly visible in the ventral pharyngeal region and appeared markedly larger than their thoracic thymus (Figure S2d).

\subsection{Conserved thymopoiesis between mouse and naked mole rat}

Next, we performed whole thymus CITE-Seq from two 3-month-old and two 12-month-old mice versus two 3-year-old and two 11-year-old NMRs (Figure S3a). The classical thymus-specific FACS pattern of $\mathrm{CD}^{+} / \mathrm{CD}^{+}$double-positive (DP) thymocytes in mice (Figure S3b) was resolved into 13 cell states of 20019 single-cell transcriptomes (Figure 2a). We captured sorted naked mole-rat $\mathrm{CD}_{3} 4^{+}$thoracic thymus cells (Figure S3c) and two LNs from the younger animals and integrated those with naked mole-rat thoracic and cervical thymi. The integrated dataset comprised 70928 cells across 11 droplet libraries from 3 tissues and one FACS-purified sample (Figure $2 b$ ). We found 15 distinct communities, which were annotated based on canonical marker gene expression for respective TC subsets (Figure 2c). The earliest developmental stage in the thymus is $\mathrm{CD}^{-} / \mathrm{CD} 8^{-}$double-negative (DN) early $\mathrm{T}$-cell progenitors (ETP), while the expression of CD44 and CD25 further subdivides the DN state. A DN2/3 cluster was the earliest distinct developmental time point detected, in mice showing exclusive CD25 $5^{\mathrm{hi}}$ expression, a Kit high-to-low-expression gradient and a fraction of CD44 ${ }^{\text {lo }}$ cells as measured by CITE signals (Figure S3d). In both species expression of PTCRA, NOTCH1 and its target HES1 were conserved in DN2/3 cells (Figure S3e-f, Table S1). The transient DN4 population ( $\left.\mathrm{CD} 44^{-} / \mathrm{CD} 25^{-}\right)$initiates TCR- $\alpha$ gene rearrangements and upregulates expression of CD4 and CD8 to yield DPs, which usually progress through an immature cycling $C D 8^{+}$intermediate single-positive population (ISP; MacDonald et al., 1988). We found an equivalent cell state DN4/ISP in both species, which was marked by overexpression of cell cycle genes, retention of PTCRA and rising expression of CD4/CD8. Remarkably, sorted CD34 ${ }^{+}$naked mole-rat thymocytes were strongly enriched for DN2/3 and DN4/ISP clusters (21\%, 35\%; Figure S3g), while CD34 mRNA and CITE signal were 
(a)

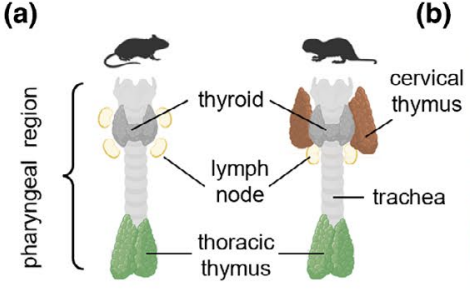

(b)

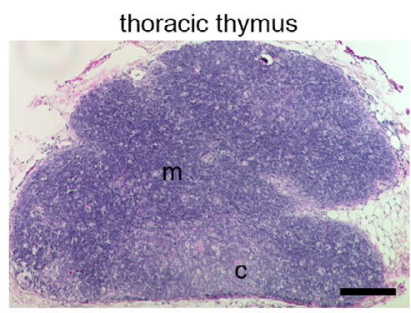

(d)

(c)

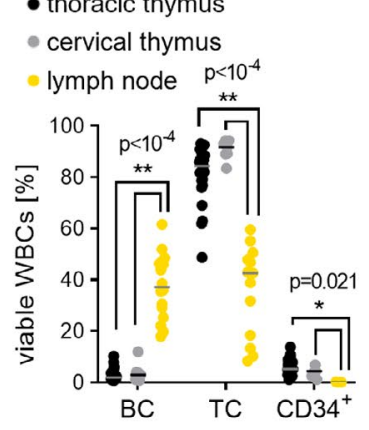

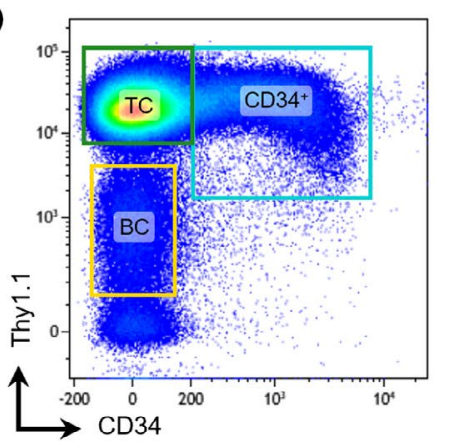

cervical thymus
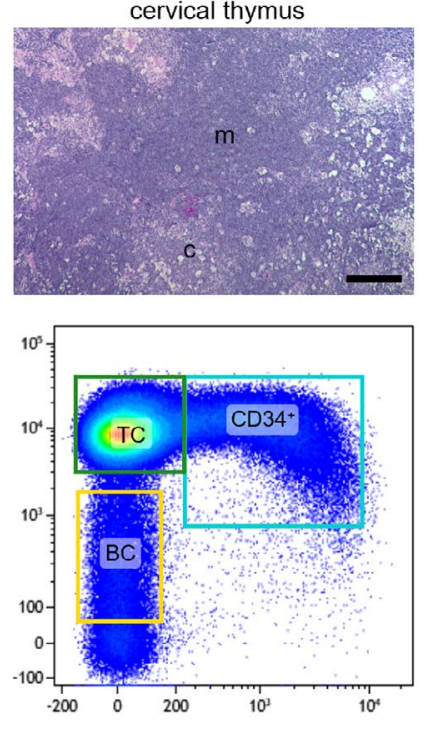

-WILEY
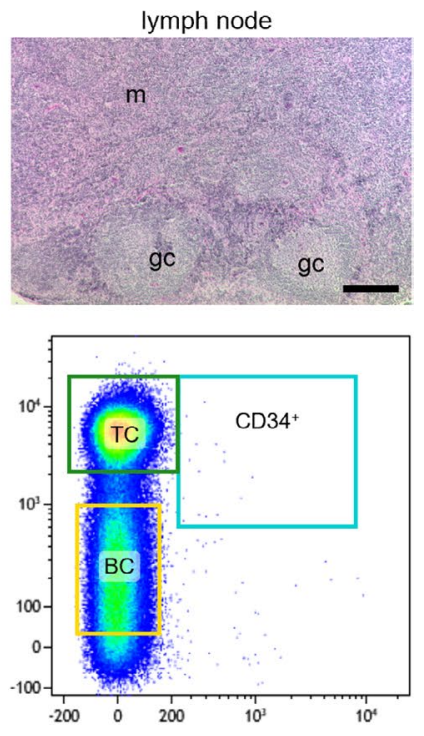

FIGURE 1 Naked mole rats have an additional thymus. (a) Schematic of the throat anatomy between mouse and naked mole rat, highlighting lymphoid organs. (b) Hematoxylin and eosin stained tissue sections of naked mole-rat thoracic [left] and cervical [middle] thymus or lymph node [right] (LN); Scale bars $200 \mu \mathrm{m}$. (c) Frequencies of cell populations across lymphatic organs. Thoracic, $n=31 ;$ cervical, $n=16$; B lymph node, $n=7$. (d) FACS gating of viable leukocytes of naked mole-rat thoracic [left] and cervical [middle] thymus or LN [right]

specific to DN2/3. This trait is shared with humans, who maintain CD34 ${ }^{+}$primitive ETPs (Terstappen et al., 1992).

Whole thymus is usually comprised of $80 \%-90 \%$ DPs (Figure S3b). Louvain clustering identified several DP communities in each species, which we labeled early (eDP) and late (IDP) according to their CD4/CD8 transcript levels. In NMRs, an additional DP cluster was formed, which was detected to $6 \%$ in LNs and thus named DP.ext (extrathymic). Total DP cell frequencies were $85 \%$ in mice and $64 \%$ in naked mole rats (Figure 2d). Expression of Rag1, anti-apoptotic Dek and Themis, indispensable for proper positive and negative TC selection in mice (Johnson et al., 2009), was conserved in eDPs, whereas IDPs activated Tox, Helios, and Gata3 transcription (Figure S3d-e). Strikingly, cell type distributions between the two NMR thymus types were identical (Figure S3h), supporting evidence was obtained from quantitation of Thy $1.1^{\text {int }} / \mathrm{CD} 34^{+}$and TC FACS populations, showing no difference between thymus origins (Figure 1c). Conversely, BCs are prevalent in $\mathrm{LNs}$ (Figure S3h), and $\mathrm{CD} 125^{+} \mathrm{BC}$ frequencies are higher in LNs than in BM, spleen, or thymi (Figure S4a,b), suggesting canonical B-lymphopoietic functions in peripheral LNs.

In summary, we found an additional pair of functional thymi in naked mole rats. Early and intermittent steps of T-lineage development appear to be conserved; however, there is a stark decrease of naked mole-rat DP proportions compared to mice.

\section{2 | Cryptic $\gamma \delta$ T-lymphocytes with a killer cell signature in naked mole rats}

Commitment toward the $\gamma \delta \mathrm{TC}$ lineage is instructed by T-cell receptor (TCR) signal strength, whereby PTCRA indicates weak and TCR $\gamma \delta$ strong signals at the DN1 stage (Munoz-Ruiz et al., 2017). We found the constant region of the TCR $\gamma$ chain (TRGC2) marking marrow ETPs (Emmrich et al., 2019); hence, the most primitive thymic partition across mouse and naked mole-rat showed conserved TRGC2 overexpression (Figure 3a). Mature murine $\gamma \delta \mathrm{TCs}$ are enriched for TRGC2. In naked mole rats, however, several mature subsets contain fractions of TRGC2-expressing cells. JAML has been shown to induce $\gamma \delta T C$ activation (Witherden et al., 2010); conversely, the mouse thymic $\gamma \delta T C$ cluster showed highest JAML expression (Figure S3f). However, we did not detect a separate $\gamma \delta T C$ population in the naked mole-rat dataset, although it had 3.5-fold more cells due to the additional cervical thymi and LNs, thereby increasing clustering resolution. Intriguingly, JAML is one of the top DN cell markers in naked mole rats and overexpressed in CD8-TCs (Figure S3f). A CD4 ${ }^{+}$cluster comprising cytotoxic T-lymphocytes (CTL), specific for GZMA and NKG7, had a cell fraction positive for TRGC2 and JAML. Interestingly, TRGC2/NKG7 co-expressing cells were also found in CD8-TCs (Figure S4c,d). We therefore compared the specific cluster markers of mouse $\gamma \delta T C$ with naked mole-rat CD4-CTL (Figure 3b), revealing a strong correlation in a shared subset of genes encompassing S100A4/10/11, RORA, IL7R, and CD44 (Table S2). Conclusively, putative naked mole-rat $\gamma \delta$ TCs appeared overtly as CTLs, similar to a human $\mathrm{V} \gamma 9 \mathrm{~V} \delta 2^{+} / \mathrm{CD} 45 \mathrm{RA}^{+} / \mathrm{CD} 27^{-}$effector memory $\gamma \delta \mathrm{TC}$ in LNs (Caccamo et al., 2005). By contrast, an abundance of murine $\gamma \delta T C$ subsets with immunomodulatory functions through mainly secreting either IFN- $\gamma$ of II-17a, but no CTLs, has been described (Pang et al., 2012). We added further evidence to this by integration of thoracic thymi scRNA-Seq from mouse and naked mole rats, encompassing 48061 cells across 10871 common genes (Figure 3c). Differential abundance quantitation confirmed the between-species bias in thymic cell composition, with all mature TC clusters significantly more frequent in naked mole rats (Figure 3d). Strikingly, the 
(a)

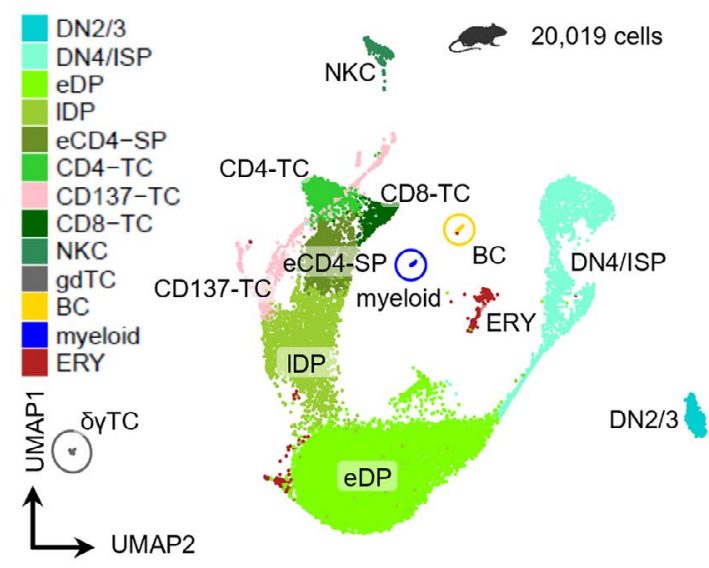

(b)

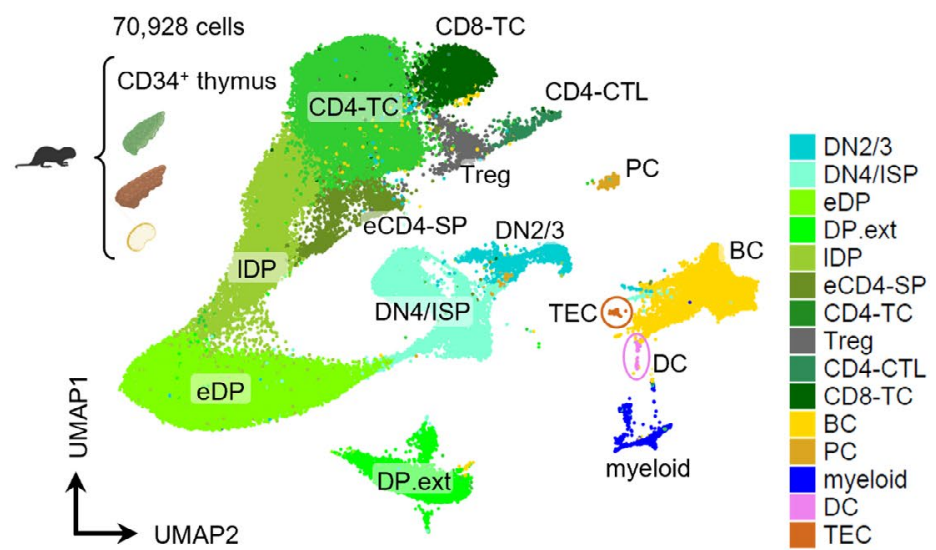

(c)

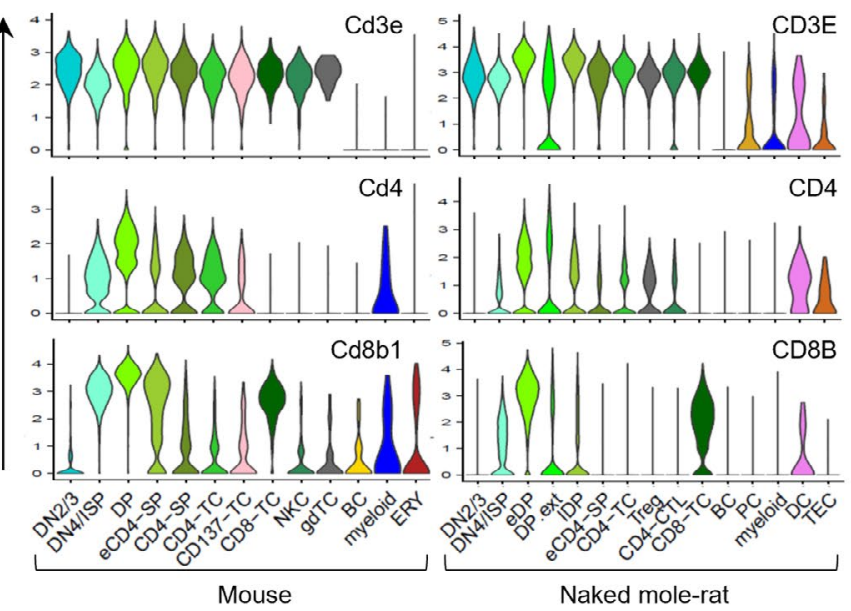

(d)

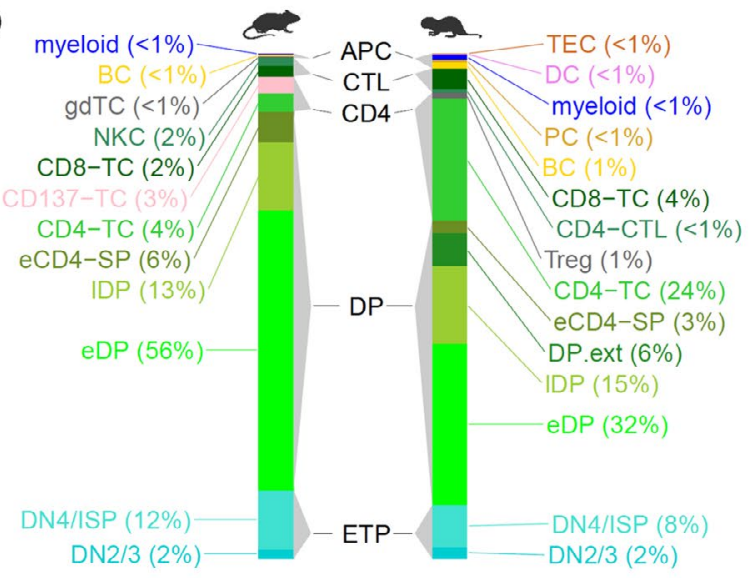

FIGURE 2 Conserved thymopoiesis between mouse and naked mole rat. (a) UMAP of scRNA-Seq from unfractionated thymus of

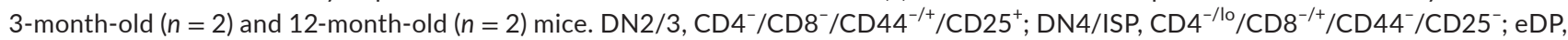
early double-positive $\mathrm{CD}^{+} / \mathrm{CD}^{+}$; IDP, late double-positive; eCD4-SP, early CD4 single-positive; CD4-TC, CD4 ${ }^{+}$T-lymphocyte; CD137-TC, $\mathrm{CD}^{+} / \mathrm{CD} 137^{+}$T-lymphocyte; CD8-TC, CD8 ${ }^{+}$T-lymphocyte; NKC, natural killer cell; gdTC, $\gamma \delta$ T-lymphocyte; BC, B-lymphocyte; myeloid, CD4 ${ }^{+}$T-lymphocyte; ERY, erythroid cells. (b) UMAP of integrated CITE-Seq from naked mole-rat thoracic $(n=4)$ and cervical thymus $(n=4)$, $\mathrm{LN}(n=2)$ and sorted CD34 ${ }^{+}$thymocytes $(n=1)$. DP.ext, extrathymic/circulating DPs; Treg, regulatory T cell; CD4-CTL, CD4 ${ }^{+}$cytotoxic Tlymphocyte; PC, plasma cell; DC, dendritic cell; TEC, thymic epithelial cell. (c) RNA expression across clusters for the mouse [left] and naked mole rat [right] CD3, CD4 and CD8B orthologs. (d) Bar chart of average cluster frequencies across species; mouse thymus, $(n=4)$; naked mole-rat thoracic thymus, $(n=4)$

integrated NK/CTL cluster mapped to mouse NKCs and $\gamma \delta T C s$, which did not co-cluster in the mouse-only dataset, and crossmapped to naked mole-rat CD4-CTLs containing the putative killer cell $\gamma \delta$ TCs (Figure 3e,f). Moreover, by mapping the constant TCR chain region transcripts from all TCR loci using the most recent NMR genome (Zhou et al., 2020), we performed absolute copy number qPCR in sorted PB-TCs and saw significantly less TRGC1/2 expression in naked mole rats (Figure S4e). Therefore, NMRs have a cryptic $\gamma \delta T C$ population with a CTL expression signature in the thymus, resembling human effector memory $\gamma \delta T C s$ with killer cell function. Unlike their $\alpha \beta T C$ counterparts that require peripheral activation for effector cell differentiation, $\gamma \delta T C s$ can be "developmentally programmed" in the thymus to generate discrete effector subsets with distinctive molecular signatures (Munoz-Ruiz et al., 2017). Our data indicate that NMR $\gamma \delta T C s$ are overtly programmed toward CTLs, potentially compensating the lack of NKCs and a diminished CD8-TC subset. $\mathrm{CD}^{+} / \mathrm{CTLA} 4^{+} / \mathrm{CD} 25^{+}$regulatory $\mathrm{T}$ cells (Treg) comprised $\sim 4 \%$ in NMR thymus and $11 \%$ in lymph nodes (Figure $\mathrm{S} 3 \mathrm{H}$ ), compared to $1 \%$ of human PB-WBCs or $3 \%$ of mouse lymph node cells (Greer et al., 2019), an adaptation in line with an expanded CD4compartment across all hematopoietic tissues (Emmrich et al., 2019).

\section{3 | No signs of thymic immunosenescence in middle-aged naked mole rats}

In mammals, thymus tissue weight decreases with age, largely by decomposition of the thymic niche required for proper TC development, wherein thymic epithelial cells (TECs) and other stromal components are replaced by adipocytes (Palmer, 2013). Mice showed a continuous decline in thymus cellularity, which is proportional to tissue weight loss, from 3 months up to 2 years of age (19-fold, 
(a)

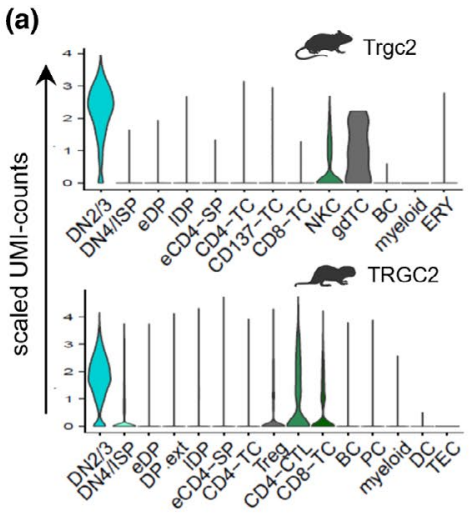

(b)

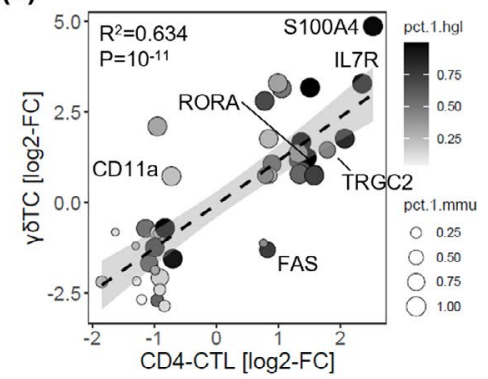

(c)

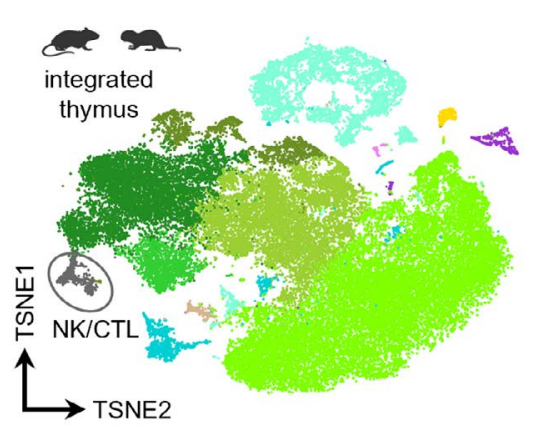

(d)

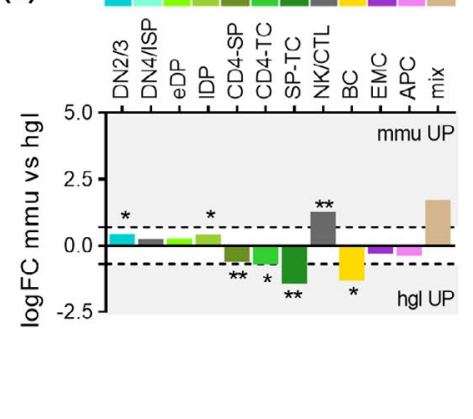

(e)

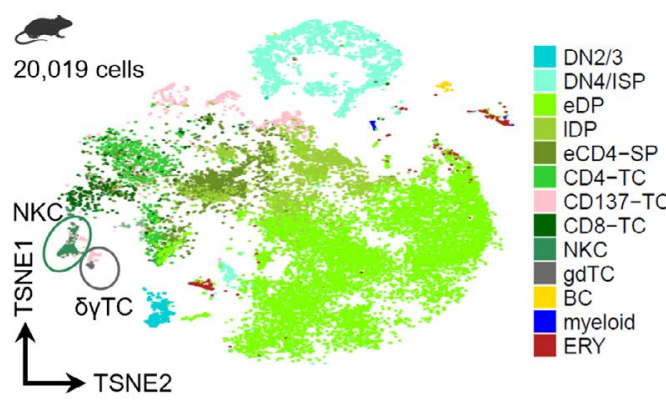

(f)

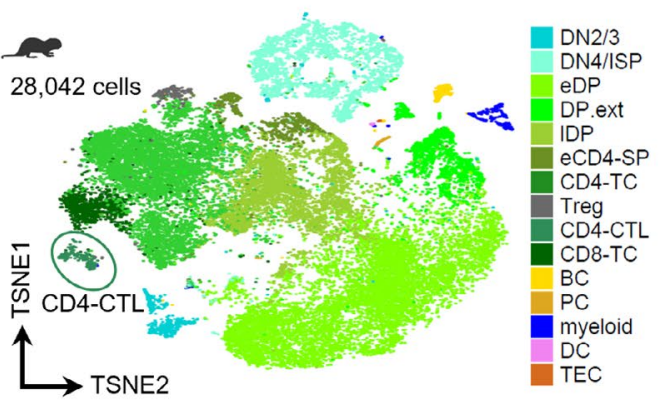

FIGURE 3 Cryptic $\delta \gamma T$ cells with killer cell signature in naked mole rats. (a) Expression of TRGC2 orthologs across cell types in thoracic thymi $(n=4)$ of mouse [top] and naked mole rat [bottom]. (b) Correlation analysis by 45 common ortholog markers of mouse $\gamma \delta T C s$ and naked mole-rat CD4-CTLs; log2-FC, $\log _{2}$ [fold-change] vs all other clusters in that species; fitted linear model y x. Pct.1.hgl, \% of CD4CTLs expressing naked mole-rat ortholog; pct.1.mmu, \% of $\gamma \delta T C$ s expressing mouse ortholog. (c) T-SNE of SCTransform-integrated mouse $(m m u, n=4)$ and naked mole rat $(h g l, n=4)$ thoracic thymi, colorbar legend for species-integrated clusters below, encircled coordinates for NK/CTL. SP-TC, single-positice T cell; EMC, erythromyeloid cell; mix, co-clustered mmu erythroid with hgl myeloid cells and DPs. (d) Differential cell type abundance across species; dotted lines, twofold change. SP-TC, NK/CTL, $p<10^{-3}$; CD4-SP, $p=0.0037$; IDP, $p=0.014$; $\mathrm{BC}, p=0.038$; CD4-TC, $p=0.041$; and DN2/3, $p=0.046$. T-SNE from species-integration for the (e), mouse or (f), naked mole-rat partition; cluster annotation and coloring from the single-species analysis

$3 \mathrm{~m}$-median $73 \times 10^{6}$ vs $24 \mathrm{~m} 3.9 \times 10^{6}$ cells; Figure $4 \mathrm{a}$ ), as reported earlier (Sempowski et al., 2002). To our surprise, we saw a significant increase in NMR thymus cellularity between 3 and 11 years of age, for both thoracic and cervical thymi. Interestingly, cervical thymi were found to contain 10-fold more thymocytes than thoracic counterparts, which was also evident from visibly smaller thoracic lobe sizes as seen above in neonates (Figure S4f). However, while murine thymus volumes strongly diminished between 3 and 12 months of age, there was no shrinkage in NMR thymi in older specimen despite the 8 year age difference. We further observed cervical LNs from either species to retain size and cellularity over aging, but while LN cell numbers were consistent even between species (Figure S4g), NMR LNs had a much larger volume than those of mice.

We next probed several correlates of thymic aging and its concomitant loss of T-lineage potential. High autoimmune regulator gene (AIRE) activity of cortical and medullary TECs orchestrates autoantigen presentation during negative selection, and its thymic expression pattern is proportional to thymic involution (Perniola, 2018). As expected, mouse AIRE levels decreased with age as measured by absolute qPCR from whole thymi, whereas NMR AIRE was consistently expressed throughout age in naked mole rats (Figure 4b). FOXN1 is the major ontogenic thymus marker by regulating TEC development and function from prenatal stage until after birth. In rodents referred to as the "nude locus," genetic disruption of Foxn1 causes athymia and hairlessness in mice and rats, and a number of TC immunodeficiency syndromes have been linked to the human ortholog (Romano et al., 2013). In mice, Foxn1 levels sharply decline in the postnatal thymus; on the contrary, NMR FOXN1 remains at the same expression level as detected in neonates, a clear neotenic feature (Emmrich et al., 2019; Figure 4c). It is important to note that both AIRE and FOXN1 expressions in NMR neonates were lower by 1-2 orders of magnitude than in mouse neonates, but whether this difference pertains to less TECs or less AIRE/FOXN1 in similar number of TECs between species warrants further investigation. We detected a TEC population in NMR thymi by scRNA-Seq with pronounced AIRE expression, whereas the mouse dataset did not feature a distinct TEC cluster (Figure S3d,e). Another hallmark of immunosenescence is the steady decline in frequency and functionality of DN early thymic progenitors in mice (Min et al., 2004). We showed that the most primitive NMR thymic progenitor compartment is $\mathrm{CD} 34^{+}$and thus quantified this fraction in thymi across an 11yr timespan. Remarkably, neither thoracic nor cervical thymi CD34 ${ }^{+}$populations diminished during this period (Figure $4 d$ ), albeit both organs showed a slight trend toward reduction, which likely becomes significant in animals aged $>20$ years. The pronounced 

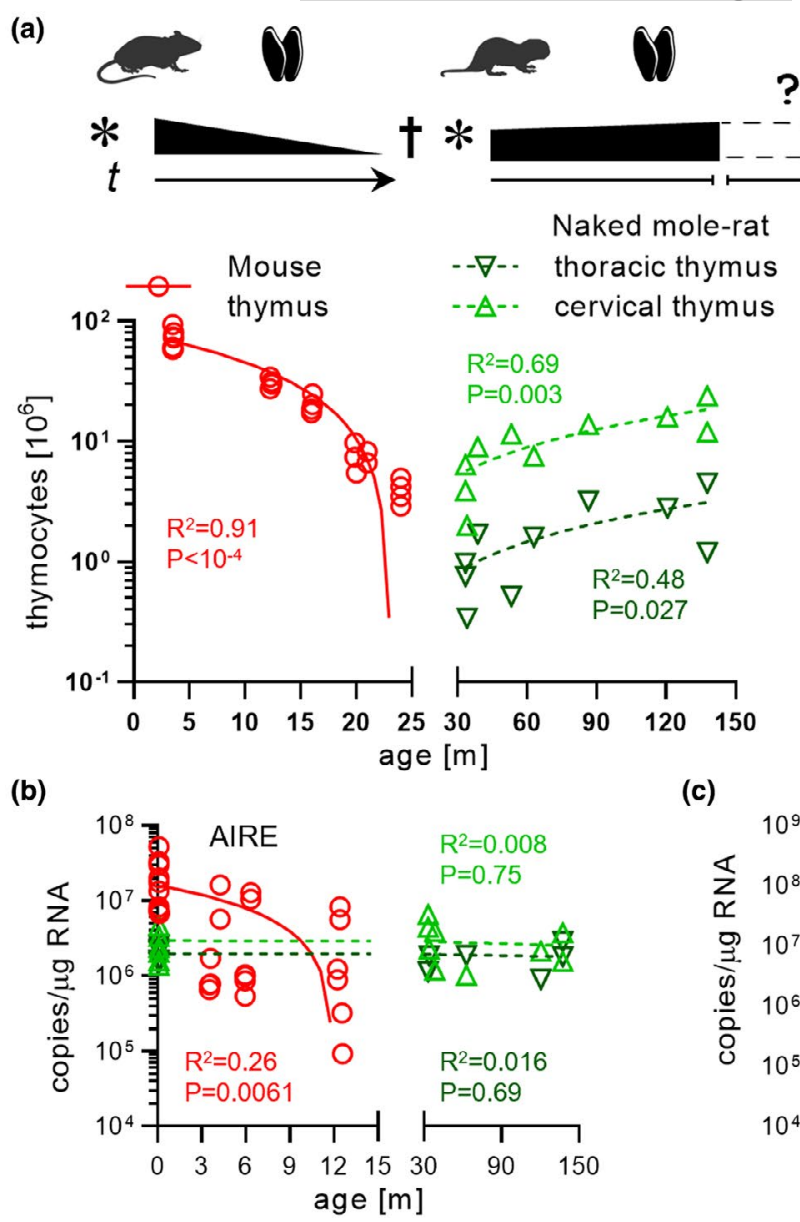

(c)

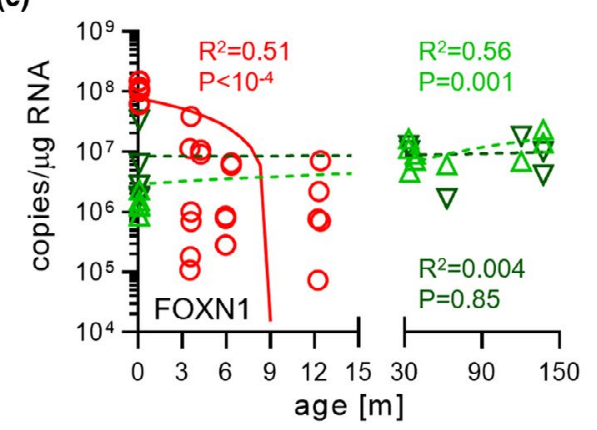

(d)
CD $34^{+}$cervical thymus

$100] \quad R^{2}=0.06$

20 上 $\quad \begin{aligned} & R^{2}=0.06 \\ & P=0.295\end{aligned}$

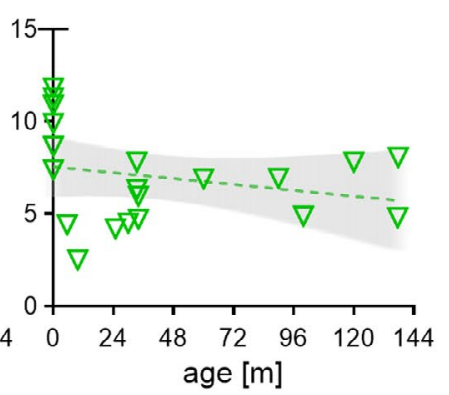

(e)

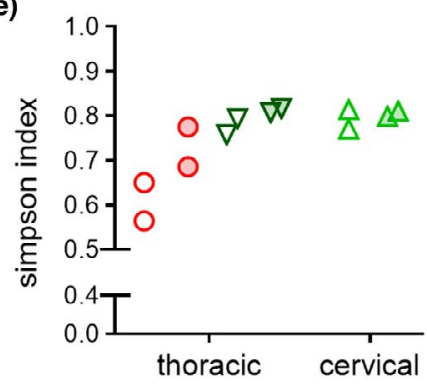

FIGURE 4 No signs of thymic immunosenescence in middle-aged naked mole rats. (a) Cellularity of mouse $(n=24)$ and naked mole-rat thoracic $(n=10)$ and cervical $(n=10)$ thymi; $\mathrm{R}^{2}$ and $p$-value derive from linear regression. Pictograms: * birth; $\dagger$, death; $t$, time. Colors and symbols used throughout the Figure. Absolute copy number determination for (b), AIRE or (c), FOXN1 ortholog mRNA in whole mouse $(n=28)$ and naked mole-rat thoracic $(n=12)$ and cervical $(n=15)$ thymi. $R^{2}$ and $p$-value derived from linear regression. (d) FACS-measured $\mathrm{CD}_{3}{ }^{+}$cell frequencies of thoracic [left, $n=53$ ] and cervical [right, $n=20$ ] thymi across age; linear regression with $95 \% \mathrm{Cl}$ as trend line; $p<0.05$, significance. (e) Simpson index of cell type diversity for whole thymus single-cell transcriptomes of 3-month- (clear circles) and 12-month-old (filled circles) mice vs 3-year- (clear triangles) and 11-year-old (filled triangles) naked mole rats

effect of thymic aging on the thymocyte pool is the dramatic decrease of naive TCs with an accompanied accumulation of effector and memory subsets in the aged thymus. Although we did not further subset clusters due to lack of established naked mole-rat markers for memory/effector vs naïve, the Simpson index as a measure of species diversity in ecosystems can be used to score fluctuations in cell type frequencies over age (Ferrall-Fairbanks \& Altrock, 2021), where represents infinite diversity and 0 , no diversity. In the naked mole rat, the Simpson index remained close to 0.8 between age groups spanning 8 years, regardless thymus type (Figure $4 \mathrm{e}$ ), while in mice it rose from 0.61 to 0.73 between age groups covering 9 months. Per cell type differential abundance quantitation showed a fivefold increase of BCs $(p=0.006)$ and 45 -fold increase of $\gamma \delta \mathrm{TCs}(p=0.011)$ in older mice, with 5 further cell types changing $>1.5$-fold (Figure S4h). In contrast, no significant up- or down-regulated cell types were found and only 3 cell types were changed by $>1.5$-fold in naked mole rats (Figure S4i). We further found that CD4 and CD8A mRNA levels were as consistent across age as FOXN1 and AIRE in naked mole-rat thymi (Figure
S4j-k). These results show that in the naked mole-rat thymocyte pool composition and transcriptional patterns are maintained for over a decade, whereas in mice changes on the cellular level coincide with onset of functional thymic regress as early as within 1 year of life.

\section{3 | DISCUSSION}

Here, we provide the first characterization of the naked mole rat thymus. We discovered that naked mole rats have an additional pair of cervical thymi. This is an unexpected finding as mammals, including humans and mice, as a rule, have only one bilateral thymus. Cervical thymi can occasionally be detected in mice, but their frequency is rare and they have unilateral appearance (Dooley et al., 2006). Similarly, rare ectopic cervical human thymi had been reported in children (Ahsan et al., 2010). In contrast, cervical thymi are a principal component of NMR ontogenesis. Interestingly, among vertebrates, chickens have seven, sharks five, and amphibians three 
thymi (Boehm \& Bleul, 2007). It is tempting to speculate that the presence of additional thymi in the naked mole rat may contribute to prolonged maintenance of immune function during their lifespan.

The ectopic thymus reflects a failed migration of thymic tissue from the third pharyngeal pouch endoderm during organogenesis, which can be found at any level of the pathway of normal thymic descent, from the angle of the mandible to the superior mediastinum (Saggese et al., 2002). It is possible that the naked mole-rat thymic anlage splits during migration and one remains in the throat. Alternatively, their parathyroid glands may have been repurposed to cervical thymi, which is less likely due to presence of a conserved PTH ortholog in naked mole-rat genome assemblies. Another explanation could be alterations as seen in ephrinB2 mutants (Gordon \& Manley, 2011), wherein the thymus remains in the anterior pharyngeal region.

We provide evidence for a delay of thymic involution in naked mole rats beyond the 1st decade of their lifespan. Age-associated marker expression and thymic cell composition remained at the level of neonates. The absence of thymic involution up to midlife is unprecedented in mammals. This would translate into similar or even slightly heightened thymic weights and cell counts for humans in their 30's. Thymic involution decreases output of naive T cells and reduces the ability to mount protective responses against new antigens. In naked mole rats, we did not see thymic involution in animals $>10$ years old, while markers for thymic function and development, AIRE and FOXN1, were maintained at neonatal levels. Furthermore, the reduction of ETPs accompanying age-related lymphoid decline did not manifest in naked mole rats, arguing that their intrinsic myeloid bias in the marrow does not predispose HSPCs toward less lymphoid commitment (Emmrich et al., 2019). However, naked mole rats are not immortal and do show frailty in old age (Edrey et al., 2011). Therefore, an eventual decline in thymic cellularity and immune function is to be anticipated, albeit delayed as opposed to the lifelong steady decline in humans and mice.

Neoteny refers to retention of juvenile phenotypes in adult organisms, hence considering humans neotenic apes (Bufill et al., 2011). NMRs feature an array of neotenic traits (Skulachev et al., 2017), including aspects of their hematopoietic system (Emmrich et al., 2019). Here, we found developmental FOXN1 and age-associated AIRE mRNA levels with little to no changes between neonate and 11-year-old adult animals. Similarly, thymic ETPs remain at neonate frequencies in NMRs. Thymic involution occurs in almost all vertebrates (Shanley et al., 2009); hence, neotenic retention of a juvenile thymus in mature, aged animals represents a likely function of longevity by maintenance of youthful TC-mediated immune function during adulthood.

\section{4 | EXPERIMENTAL PROCEDURES}

\subsection{Animals}

Ethical and legal approval was obtained prior to the start of the study by the University of Rochester Committee on Animal Resources (UCAR). All animal experiments were approved and performed in accordance with guidelines instructed by UCAR with protocol numbers 2009-054 (naked mole rat) and 2017-033 (mouse). Naked mole rats were from the University of Rochester colonies, housing conditions as described (Ke et al., 2014). C57BL/6 mice were obtained from NIA.

\section{2 | Primary cell isolation}

Marrow from mice and naked mole rats was extracted from femora, tibiae, humeri, iliaci, and vertebrae by crushing. Thymus and lymph nodes were minced over a $70 \mu \mathrm{m}$ strainer and resuspended in FACS buffer. Blood from mice was drawn via retroorbital capillary bleeding; naked mole-rat blood was obtained via heart puncture.

\section{3 | Histology}

Imaging and analysis was performed using a using a Nikon Eclipse Ti-S microscope. Coverslips were applied with DEPEX Mounting media (Electron Microscopy Sciences), except for Alkaline Phosphatase staining where Vectashield Hard Set Mounting Medium for Fluorescence (Vector) was applied. Soft tissues were stored in 10\% neutral buffered formalin, processing was done using a Sakura Tissue-Tek VIP 6 automated histoprocessor, and paraffin embedding was done using a Sakura Tissue-Tek TEC 5 paraffin embedding center. A Microm HM315 microtome was used to section tissues at a thickness of $5 \mu \mathrm{m}$, which then were floated onto a slide with a water bath at a temperature between 45 and $55^{\circ} \mathrm{C}$. Sections were deparaffinized and rehydrated to distilled water through xylene and graded ethanol (100\%-70\%).

\subsubsection{Hematoxylin and eosin}

Sections were stained with Mayers Hematoxylin (Sigma) for 1 min and washed with tap water to remove excess blue coloring. Soft tissue sections were further decolorized with 3 dips in $0.5 \%$ acid alcohol and washed in distilled water. The nuclei of sections were blued in $1 \mathrm{X}$ PBS for $1 \mathrm{~min}$ and washed again in distilled water. An alcoholic-eosin counterstain was applied for $30 \mathrm{~s}$ before slides were immediately dehydrated and cleared through 3 changes of $95 \%$ ethanol, 2 changes of $100 \%$ ethanol, and three changes of Xylene for 1 min each.

\subsection{2 | Cytokeratin}

Paraffin sections ( $4 \mu \mathrm{m}$ thick) of FFPE thymus and lymph node tissues (NMR, mouse, and human control) were stained for cytokeratin (AE1/AE3, Dako GA05361-2) on a Dako Omnis autostainer with pressure cooker antigen retrieval (TrisEDTA; $\mathrm{pH}$ 9). A section of normal human thymus resected for routine clinical care at the university of Rochester medical center was stained for $H \& E$ and cytokeratin for morphological comparison. The human, mouse, and NMR samples were stained in the same run on the same machine. 


\section{4 | Flow Cytometry}

Flow cytometry analysis was performed at the URMC Flow Core on a LSR II or LSRFortessa (both BD), or on our laboratories CytoFlex S (Beckman Coulter). Kaluza 2.1 (Beckman Coulter) was used for data analysis. Staining and measurement were done using standard protocols. Red blood cell lysis was done by resuspending marrow pellets in $4 \mathrm{ml}$, spleen pellets in $1 \mathrm{ml}$ and up to $500 \mu \mathrm{l} \mathrm{blood}$ in $20 \mathrm{ml}$ of RBC lysis buffer, prepared by dissolving $4.1 \mathrm{~g} \mathrm{NH}_{4} \mathrm{Cl}$ and $0.5 \mathrm{~g} \mathrm{KHCO}_{3}{ }^{-}$into $500 \mathrm{ml}$ double-distilled $\mathrm{H}_{2} \mathrm{O}$ and adding $200 \mu \mathrm{l} 0.5$ M EDTA. Marrow and spleen were incubated for $2 \mathrm{~min}$ on ice; blood was lysed for $30 \mathrm{~min}$ at room temperature. Cells were resuspended in FACS buffer (DPBS, 2mM EDTA, 2\% FBS [Gibco]) at $1 \times 10^{7}$ cells $/ \mathrm{ml}$; antibodies were added at $1 \mu \mathrm{l} / 10^{7}$ cells, vortexmixed and incubated for $30 \mathrm{~min}$ at $4^{\circ} \mathrm{C}$ in the dark. DAPI (Thermo Fisher) @ $1 \mu \mathrm{g} / \mathrm{ml}$ was used as viability stain. The primary gating path for all unfixed samples was as follows: scatter-gated WBC (FSC-A vs SSC-A) => singlets1 (SSC-W vs SSC-H) $=>$ singlets 2 $($ FSC-W vs FSC-H) $=>$ viable cells (SSC vs DAPI) $==$ proceed with specific markers/probes. Compensation was performed using fluorescence minus one (FMO) controls for each described panel. For antibody validation, we incubated 1 mio cells in $100 \mu \mathrm{l}$ Cell Staining Buffer (BioLegend; Cat\# 420201) and added $5 \mu$ l Human TrueStain $\mathrm{FCX}^{\mathrm{TM}}$ and $0.5 \mu \mathrm{l}$ TruStain FcX ${ }^{\mathrm{TM}}$ PLUS, followed by incubation for $10 \mathrm{~min}$ at $4^{\circ} \mathrm{C}$. We then proceeded with fluorescent antibody staining as above.

Immunophenotyping of naked mole-rat BM, spleen, thymus, PB, and lymph nodes: CD90 FITC; CD125 PE; Thy1.1 PE-Cy7; CD34 APC; and CD11b APC-Cy7. Quantification of murine BM SLAM HSCs was performed using mouse LIN Pacific Blue; Sca-1 BUV395; CD150 PE; Kit PE-Cy7; CD48 APC-Cy7. Quantification of human BM LT-HSCs was performed using human LIN Pacific Blue; CD34 APC; CD38 APC-Cy7; CD45RA FITC; and CD90 PE-Cy7. Fluorescence minus one (FMO) controls were applied for fluorescent spillover compensations for each species and tissue used.

Sorting was performed at the URMC Flow Core on a FACSAria (BD) using a $85 \mu \mathrm{m}$ nozzle; staining was done as described. Human HSCs were sorted for population RNA-Seq as $\mathrm{LIN}^{-} / \mathrm{CD} 34^{+} / \mathrm{CD} 38^{\mathrm{Lo}} /$ $\mathrm{CD} 4 \mathrm{RA}^{-} / \mathrm{CD}^{\mathrm{Dim}}$ (Figure S5A). Naked mole-rat HSPC populations were sorted as described with a lineage cocktail comprised of CD11b, CD18, CD90, and CD125 (NMR LIN). Naked mole-rat marrow and spleen sorting panel was as follows: NMR LIN Pacific Blue; Thy1.1 PE-Cy7; CD34 APC. Naked mole-rat blood sorting panel was as follows: Thy1.1 PE-Cy7; CD11b APC-Cy7.

\subsection{Quantitative PCR}

Mouse and naked mole-rat sorted TCs and thymic tissue were used for RNA extraction by Trizol (Thermo Fisher). RNA was quantified using a NanoDrop One (Thermo Fisher), and 100 ng was used as input for the High Capacity cDNA Reverse Transcription Kit (Thermo Fisher). RT reaction was performed according to instructions and the
$20 \mu \mathrm{l}$ reaction diluted to $200 \mu \mathrm{l}$, of which $5 \mu \mathrm{l}$ was used per qPCR reaction. We used iTaq Universal SYBR Green Supermix (Bio-Rad) on a CFX Connect ${ }^{\circledR}$ RealTime System (Bio-Rad) with a three-step cycling of $10 \mathrm{~s} 95^{\circ} \mathrm{C}, 20 \mathrm{~s} 60^{\circ} \mathrm{C}, 30 \mathrm{~s} 72^{\circ} \mathrm{C}$ for 40 cycles. All primers (IDTDNA) were validated to amplify a single amplicon at the above PCR conditions by gel electrophoresis. Gene sequences for primer design by Primer3Plus were retrieved from ENSEMBL, with the exception of the $\mathrm{T}$-cell receptor $\mathrm{C}$-region genes for naked mole rat. Here, we used the WBM RNA-Seq from the transcriptome assembly below to map those genes in a recently published naked molerat genome (Zhou et al., 2020) using Apollo software and custom scripts. For absolute copy number quantitation, qPCR amplicons were gel-purified using the QIAquick Gel Extraction Kit (Qiagen) and subcloned into the pCR2.1 plasmid using the TOPO-TA cloning Kit (Thermo Fisher). Plasmids were prepared using the QIAprep Spin Miniprep Kit (Qiagen). Sanger sequencing was performed by Genewiz using M13 forward and reverse primers. Standard curves were prepared across a 10 -fold dilution range from 20ag to $20 \mathrm{pg}$ of plasmid DNA. All amplicon gel images, amplicon plasmids, and standard curve data are available upon request.

\section{6 | Single-cell RNA-Seq}

10,000 $\mathrm{DAPI}^{-}$thymus cells from 2 mice aged 3 months (o $\& 0^{\top}$ ) and 2 mice aged 12 months ( $\&_{\&} 0^{\top}$ ), or 2 naked mole rats aged 3 years $\left(\$ \& ठ^{\top}\right)$ and 3 naked mole rats aged 11 years ( $\left.\$ \& 0^{\star}\right)$, were subjected to CITE-Seq with $10 \mathrm{X} v 3$ chemistry. In addition, for all naked molerat specimen we collected 10,000 $\mathrm{DAPI}^{-}$cervical thymus cells. For 2 naked mole rats aged 3 years ( $\& \& 0^{\star}$ ), we collected 10,000 $\mathrm{DAPI}^{-}$cells from cervical lymph nodes. Cells were processed for TotalSeq $^{\text {TM }}$ CITE reagents according to the manufacturers' instructions (BioLegend), using both human and mouse Fc blocking reagents (BioLegend). Cellular suspensions were loaded on a Chromium Single-Cell Instrument (10x Genomics) to generate single-cell Gel Bead-in-Emulsions (GEMs). Single-cell RNA-Seq libraries were prepared using Chromium Next GEM Single Cell 3' GEM, Library \& Gel Bead Kit v3.1 (10x Genomics). The beads were dissolved, and cells were lysed per manufacturer's recommendations. GEM reverse transcription (GEM-RT) was performed to produce a barcoded, full-length cDNA from poly-adenylated mRNA. After incubation, GEMs were broken and the pooled post-GEM-RT reaction mixtures were recovered and cDNA was purified with silane magnetic beads (DynaBeads MyOne Silane Beads, PN37002D, ThermoFisher Scientific). The entire purified post-GEM-RT product was amplified by PCR. This amplification reaction generated sufficient material to construct a 3' cDNA library. Enzymatic fragmentation and size selection was used to optimize the cDNA amplicon size, and indexed sequencing libraries were constructed by End Repair, Atailing, Adaptor Ligation, and PCR. Final libraries contain the P5 and $\mathrm{P} 7$ priming sites used in Illumina bridge amplification. In parallel, CITE-seq library amplification is performed following SPRI bead purification of CITE-seq cDNA using Q5 Hot Start HiFi Master Mix 
(New England Biolabs), SI PCR primer (IDT), and indexed TruSeq Small RNA PCR primers (Illumina) as specified (Stoeckius et al., 2017). Amplified CITE-seq libraries are purified using AMPure XP (Beckman Coulter) beads and quantified by Qubit dsDNA assay (ThermoFisher) and Bioanalyzer HSDNA (Agilent) analysis. CITEseq libraries were pooled with 10x Genomics gene expression libraries for sequencing on Illumina's NovaSeq 6000. Barcodes were quality filtered to keep cells between 200-5000 detected genes/cell and $<20,000$ counts per cell. RNA assay was lognormalized with "scale. factor = 1 e4"; CITE assay was "CLR" normalized. Variable features were detected with arguments selection.method = "vst", nfeatures $=3000$. Canonical correlation analysis (CCA) was used to integrate libraries (Stuart et al., 2019) from either species with FindIntegrationAnchors with dims =1:50, anchor. features =3000, reduction $=$ "cca". For naked mole rat, we integrated 4 thoracic thymus, 4 cervical thymus, 2 lymph node, and $1 \mathrm{CD}^{+} 4^{+}$thymocyte libraries (Figure 2b). Scores for G2 M and S phases were obtained using Seurat Cellcyclescoring as described in the respective Seurat vignette. Clustering was done using Seurat's FindClusters function with resolution $=0.5$. Next, we used the doublet detection and removal workflow as suggested in the Bioconductor OSCA vignette. Briefly, we run findDoubletcluster from the $s c D$ bIFinder package, followed by in silico simulation of doublets from the single-cell expression profiles (Dahlin et al., 2018) using computeDoubletDensity from BiocSingular package, and excluded any cluster which was identified in both methods. The DEGs for each cluster were detected by FindAllMarkers function with arguments test.use = "MAST", logfc.threshold = $\log (2)$, min.pct $=0.25$, return.thresh $=0.05$. CITE feature/antibody marker detection was done as described for transcript cluster markers with the exception of test.use = "wilcox". The heatmap of the top overexpressed markers for each cluster in Figure S7A shows that CCA integration worked efficiently. However, cells from sorted $\mathrm{CD} 34^{+}$thymocytes of the integrated clusters $\mathrm{PC}$, and to a lesser extent eCD4-SP, feature a marker signature of combined DN2/3 and DN4/ISP clusters. We attribute this primarily to the difference in Chromium Single Cell 3' Reagent Kits (10X Genomics), which were $\mathrm{v} 3$ chemistry for all integrated lymphoid tissues, except for the sorted $\mathrm{CD} 34^{+}$thymocyte library captured with v2 chemistry. We performed manual curation of the cell type annotation based on canonical markers from the literature. Mapping of naked mole-rat $\gamma \delta \mathrm{TCs}$ to the CD4-CTL cluster was done by intersecting 620 mouse $\gamma \delta T C$ markers with 129 naked mole-rat CD4-CTL markers, yielding 45 genes in both clusters, which we regressed by a linear model from the stats package (Figure 3b). DA testing was performed as described (Lun et al., 2017). For the CCA-integrated naked mole-rat lymphoid dataset, we show cell type abundances between age groups across both thoracic and cervical thymi (Figure S4i). Regardless of DA testing across both or separate testing of either thoracic or cervical thymi, no cell type was significantly (FDR $<0.05$ ) changed. SCTransform was used to integrate scaled, clustered, and annotated mouse and naked mole-rat unfractionated thoracic thymus datasets: SelectIntegrationFeatures with nfeatures $=3000$, FindIntegrationAnchors with normalization.method = "SCT". Cell cycle scoring, clustering, and marker detection were performed as described above. Simpson's index as calculated by $D^{S}=1-\sum \frac{n_{i}\left(n_{i}-1\right)}{N(N-1)}$ using the vegan package was determined as diversity of cell types across libraries (Figure 4e).

\section{7 | Quantification and Statistical Analysis}

Data are presented as the mean \pm SD. Statistical tests performed can be found in the figure legends. $p$ values of $<0.05$ were considered statistically significant. Statistical analyses were carried out using Prism 9 software (GraphPad) unless otherwise stated.

\section{ACKNOWLEDGMENTS}

Many thanks to Cameron Baker for cellranger preprocessing of scRNA-Seq data, Michelle Zanche, Jeffrey Malik, and John Ashton for Genomic Research Core support. The authors thank the Center for Integrated Research Computing (CIRC) at the University of Rochester for providing computational resources and technical support and the URMC Flow Core for assistance with sorting and Seahorse. This work was supported by the US National Institutes of Health grants to V.G. and A.S., and V.N.G. S.E. is a fellow of HFSP.

\section{CONFLICT OF INTEREST}

The authors declare that they have no conflict of interest.

\section{AUTHOR CONTRIBUTIONS}

S.E. designed and supervised research, performed most experiments, and analyzed data; F.T.Z. performed histology quantifications, animal perfusions, and data analysis; A.T. contributed to bioinformatics analyses; X.Z. improved genome assembly; Q.Z. mapped TCR genes; M.D.G. performed histology and provided human BM specimen; E.M.I., Z.Z. and V.N.G. contributed to data analysis; A.S. and V.G. supervised research; S.E., A.S., and V.G. wrote the manuscript with input from all authors.

\section{DATA AVAILABILITY STATEMENT}

The data that support the findings of this study are available in the supplementary material and Supplementary Tables of this article. The single-cell RNA-sequencing data are available at figshare, https://doi.org/10.6084/m9.figshare.c.5624737.v2. Further information and requests for resources and reagents should be directed to and will be replied by the Lead Contact, Stephan Emmrich (Stephan-Emmrich@gmx.net).

\section{ORCID}

Stephan Emmrich (D) https://orcid.org/0000-0002-0537-4242

Vadim N. Gladyshev (D) https://orcid.org/0000-0002-0372-7016

Andrei Seluanov (D) https://orcid.org/0000-0003-3400-538X

Vera Gorbunova (D) https://orcid.org/0000-0001-8979-0333 


\section{REFERENCES}

Ahsan, F., Allison, R., \& White, J. (2010). Ectopic cervical thymus: Case report and review of pathogenesis and management. Journal of Laryngology and Otology, 124(6), 694-697. https://doi.org/10.1017/ S0022215109992180

Boehm, T., \& Bleul, C. C. (2007). The evolutionary history of lymphoid organs. Nature Immunology, 8(2), 131-135. https://doi.org/10.1038/ ni1435

Buffenstein, R. (2008). Negligible senescence in the longest living rodent, the naked mole-rat: insights from a successfully aging species. Journal of Comparative Physiology B, 178(4), 439-445. https://doi. org/10.1007/s00360-007-0237-5

Buffenstein, R., \& Jarvis, J. U. (2002). The naked mole rat-a new record for the oldest living rodent. Science of Aging Knowledge Environment: SAGE KE, 2002(21), pe7.

Buffenstein, R., \& Ruby, J. G. (2021). Opportunities for new insight into aging from the naked mole-rat and other non-traditional models. Nature Aging, 1(1), 3-4. https://doi.org/10.1038/s43587-02000012-4

Bufill, E., Agusti, J., \& Blesa, R. (2011). Human neoteny revisited: The case of synaptic plasticity. American Journal of Human Biology, 23(6), 729-739. https://doi.org/10.1002/ajhb.21225

Caccamo, N., Meraviglia, S., Ferlazzo, V., Angelini, D., Borsellino, G., Poccia, F., Battistini, L., Dieli, F., \& Salerno, A. (2005). Differential requirements for antigen or homeostatic cytokines for proliferation and differentiation of human Vgamma9Vdelta2 naive, memory and effector T cell subsets. European Journal of Immunology, 35(6), 1764-1772.

Dahlin, J. S., Hamey, F. K., Pijuan-Sala, B., Shepherd, M., Lau, W. W. Y., Nestorowa, S., Weinreb, C., Wolock, S., Hannah, R., Diamanti, E., Kent, D. G., Gottgens, B., \& Wilson, N. K. (2018). A single-cell hematopoietic landscape resolves 8 lineage trajectories and defects in Kit mutant mice. Blood, 131(21), e1-e11. https://doi.org/10.1182/ blood-2017-12-821413

de Magalhaes, J. P., Costa, J., \& Toussaint, O. (2005). HAGR: The human ageing genomic resources. Nucleic Acids Research, 33(Database issue), 537.

Delaney, M. A., Nagy, L., Kinsel, M. J., \& Treuting, P. M. (2013). Spontaneous histologic lesions of the adult naked mole rat (Heterocephalus glaber): A retrospective survey of lesions in a zoo population. Veterinary Pathology, 50(4), 607-621.

Delaney, M. A., Ward, J. M., Walsh, T. F., Chinnadurai, S. K., Kerns, K., Kinsel, M. J., \& Treuting, P. M. (2016). Initial case reports of cancer in naked mole-rats (Heterocephalus glaber). Veterinary Pathology, 53(3), 691-696.

Dixit, V. D. (2012). Impact of immune-metabolic interactions on agerelated thymic demise and T cell senescence. Seminars in Immunology, 24(5), 321-330. https://doi.org/10.1016/j.smim.2012.04.002

Dooley, J., Erickson, M., Gillard, G. O., \& Farr, A. G. (2006). Cervical thymus in the mouse. The Journal of Immunology, 176(11), 6484-6490. https://doi.org/10.4049/jimmunol.176.11.6484

Edrey, Y. H., Hanes, M., Pinto, M., Mele, J., \& Buffenstein, R. (2011). Successful aging and sustained good health in the naked mole rat: a long-lived mammalian model for biogerontology and biomedical research. ILAR Journal, 52(1), 41-53. https://doi.org/10.1093/ ilar.52.1.41

Emmrich, S., Mariotti, M., Takasugi, M., Straight, M. E., Trapp, A., Gladyshev, V. N., Seluanov, A., \& Gorbunova, V. (2019). The hematopoietic landscape at single-cell resolution reveals unexpected stem cell features in naked mole-rats. bioRxiv, 859454.

Ferrall-Fairbanks, M. C., \& Altrock, P. M. (2021). Investigating interand intrasample diversity of single-cell RNA sequencing datasets. Methods in Molecular Biology, 2194, 177-186.

Finch, C. E. (1990). Longevity, senescence, and the genome. University of Chicago Press.
Gorbunova, V., Seluanov, A., Zhang, Z., Gladyshev, V. N., \& Vijg, J. (2014). Comparative genetics of longevity and cancer: Insights from longlived rodents. Nature Reviews Genetics, 15(8), 531-540. https://doi. org/10.1038/nrg3728

Gordon, J., \& Manley, N. R. (2011). Mechanisms of thymus organogenesis and morphogenesis. Development, 138(18), 3865-3878. https://doi. org/10.1242/dev.059998

Greer, J. P., Rodgers, G. M., Glader, B., Arber, D. A., Means, R. T. Jr, List, A. F., Appelbaum, F. R., Dispenzieri, A., \& Fehniger, T. A. (2019). Wintrobe's clinical hematology. Lippincott Williams \& Wilkins (LWW).

Hilton, H. G., Rubinstein, N. D., Janki, P., Ireland, A. T., Bernstein, N., Fong, N. L., Wright, K. M., Smith, M., Finkle, D., Martin-McNulty, B., Roy, M., Imai, D. M., Jojic, V., \& Buffenstein, R. (2019). Single-cell transcriptomics of the naked mole-rat reveals unexpected features of mammalian immunity. PLoS Biology, 17(11), e3000528. https:// doi.org/10.1371/journal.pbio.3000528

Izraelson, M., Nakonechnaya, T. O., Davydov, A. N., Dronina, M. A., Miskevich, D. A., Mamedov, I. Z., Barbashova, L. N., Shugay, M., Bolotin, D. A., Staroverov, D. B., Kondratyuk, E. Y., Bogdanova, E. A., Lukyanov, S., Shams, I., Britanova, O. V., \& Chudakov, D. M. (2018). T cell immunity does not age in a long-lived rodent species. bioRxiv, 259374.

Johnson, A. L., Aravind, L., Shulzhenko, N., Morgun, A., Choi, S. Y., Crockford, T. L., Lambe, T., Domaschenz, H., Kucharska, E. M., Zheng, L., Vinuesa, C. G., Lenardo, M. J., Goodnow, C. C., Cornall, R. J., \& Schwartz, R. H. (2009). Themis is a member of a new metazoan gene family and is required for the completion of thymocyte positive selection. Nature Immunology, 10(8), 831-839. https://doi. org/10.1038/ni.1769

Ke, Z., Vaidya, A., Ascher, J., Seluanov, A., \& Gorbunova, V. (2014). Novel husbandry techniques support survival of naked mole rat (Heterocephalus glaber) pups. Journal of the American Association for Laboratory Animal Science, 53(1), 89-91.

Lewis, K. N., Rubinstein, N. D., \& Buffenstein, R. (2018). A window into extreme longevity; the circulating metabolomic signature of the naked mole-rat, a mammal that shows negligible senescence. Geroscience, 40(2), 105-121. https://doi.org/10.1007/s1135 7-018-0014-2

Lun, A. T. L., Richard, A. C., \& Marioni, J. C. (2017). Testing for differential abundance in mass cytometry data. Nature Methods, 14(7), 707709. https://doi.org/10.1038/nmeth.4295

Lynch, H. E., Goldberg, G. L., Chidgey, A., Van den Brink, M. R., Boyd, R., \& Sempowski, G. D. (2009). Thymic involution and immune reconstitution. Trends in Immunology, 30(7), 366-373. https://doi. org/10.1016/j.it.2009.04.003

MacDonald, H. R., Budd, R. C., \& Howe, R. C. (1988). A CD3- subset of CD4-8+ thymocytes: A rapidly cycling intermediate in the generation of CD4+8+ cells. European Journal of Immunology, 18(4), 519523. https://doi.org/10.1002/eji.1830180405

Min, H., Montecino-Rodriguez, E., \& Dorshkind, K. (2004). Reduction in the developmental potential of intrathymic $T$ cell progenitors with age. The Journal of Immunology, 173(1), 245-250. https://doi. org/10.4049/jimmunol.173.1.245

Munoz-Ruiz, M., Sumaria, N., Pennington, D. J., \& Silva-Santos, B. (2017). Thymic determinants of gammadelta T cell differentiation. Trends in Immunology, 38(5), 336-344.

O'Connor, T. P., Lee, A., Jarvis, J. U., \& Buffenstein, R. (2002). Prolonged longevity in naked mole-rats: age-related changes in metabolism, body composition and gastrointestinal function. Comparative Biochemistry and Physiology Part A Molecular Integrative Physiology, 133(3), 835-842. https://doi.org/10.1016/S1095-6433(02)00198-8

Palmer, D. B. (2013). The effect of age on thymic function. Frontiers in Immunology, 4, 316. https://doi.org/10.3389/fimmu.2013.00316

Pang, D. J., Neves, J. F., Sumaria, N., \& Pennington, D. J. (2012). Understanding the complexity of gammadelta T-cell subsets in mouse and human. Immunology, 136(3), 283-290. 
Perniola, R. (2018). Twenty years of AIRE. Frontiers in Immunology, 9, 98. https://doi.org/10.3389/fimmu.2018.00098

Puppione, D. L., Tran, D. P., Zenaidee, M. A., Charugundla, S., Whitelegge, J. P., \& Buffenstein, R. (2021). Naked mole-rat, a rodent with an apolipoprotein A-I dimer. Lipids, 56(3), 269-278.

Romano, R., Palamaro, L., Fusco, A., Giardino, G., Gallo, V., Del Vecchio, L., \& Pignata, C. (2013). FOXN1: A master regulator gene of thymic epithelial development program. Frontiers in Immunology, 4, 187. https://doi.org/10.3389/fimmu.2013.00187

Ruby, J. G., Smith, M., \& Buffenstein, R. (2018). Naked Mole-Rat mortality rates defy gompertzian laws by not increasing with age. eLife, 7 , e31157. https://doi.org/10.7554/eLife.31157

Saggese, D., Ceroni Compadretti, G., \& Cartaroni, C. (2002). Cervical ectopic thymus: A case report and review of the literature. International Journal of Pediatric Otorhinolaryngology, 66(1), 77-80. https://doi.org/10.1016/S0165-5876(02)00210-0

Schlenner, S. M., \& Rodewald, H. R. (2010). Early T cell development and the pitfalls of potential. Trends in Immunology, 31(8), 303-310.

Sempowski, G. D., Gooding, M. E., Liao, H. X., Le, P. T., \& Haynes, B. F. (2002). T cell receptor excision circle assessment of thymopoiesis in aging mice. Molecular Immunology, 38(11), 841-848. https://doi. org/10.1016/S0161-5890(01)00122-5

Shah, D. K., \& Zuniga-Pflucker, J. C. (2014). An overview of the intrathymic intricacies of $\mathrm{T}$ cell development. The Journal of Immunology, 192(9), 4017-4023. https://doi.org/10.4049/jimmunol.1302259

Shanley, D. P., Aw, D., Manley, N. R., \& Palmer, D. B. (2009). An evolutionary perspective on the mechanisms of immunosenescence. Trends in Immunology, 30(7), 374-381. https://doi.org/10.1016/j. it.2009.05.001

Skulachev, V. P., Holtze, S., Vyssokikh, M. Y., Bakeeva, L. E., Skulachev, M. V., Markov, A. V., Hildebrandt, T. B., \& Sadovnichii, V. A. (2017). Neoteny, prolongation of youth: From naked mole rats to "naked apes" (humans). Physiological Reviews, 97(2), 699-720. https://doi. org/10.1152/physrev.00040.2015

Stoeckius, M., Hafemeister, C., Stephenson, W., Houck-Loomis, B., Chattopadhyay, P. K., Swerdlow, H., Satija, R., \& Smibert, P. (2017). Simultaneous epitope and transcriptome measurement in single cells. Nature Methods, 14(9), 865-868. https://doi.org/10.1038/ nmeth.4380.

Stuart, T., Butler, A., Hoffman, P., Hafemeister, C., Papalexi, E., Mauck, W. M., Hao, Y., Stoeckius, M., Smibert, P., \& Satija, R. (2019). Comprehensive integration of single-cell data. Cell, 177(7), 18881902.e21. https://doi.org/10.1016/j.cell.2019.05.031

Terstappen, L. W., Huang, S., \& Picker, L. J. (1992). Flow cytometric assessment of human T-cell differentiation in thymus and bone marrow. Blood, 79(3), 666-677.
Tian, X., Azpurua, J., Hine, C., Vaidya, A., Myakishev-Rempel, M., Ablaeva, J., Mao, Z., Nevo, E., Gorbunova, V., \& Seluanov, A. (2013). High-molecular-mass hyaluronan mediates the cancer resistance of the naked mole rat. Nature, 499(7458), 346-349.

Tian, X., Azpurua, J., Ke, Z., Augereau, A., Zhang, Z. D., Vijg, J., Gladyshev, V. N., Gorbunova, V., \& Seluanov, A. (2015). INK4 locus of the tumor-resistant rodent, the naked mole rat, expresses a functional p15/p16 hybrid isoform. Proceedings of the National Academy of Sciences of the United States of America, 112(4), 1053-1058.

Tong, Q. Y., Zhang, J. C., Guo, J. L., Li, Y., Yao, L. Y., Wang, X., Yang, Y. G., \& Sun, L. G. (2020). Human thymic involution and aging in humanized mice. Frontiers in Immunology, 11, 1399. https://doi.org/10.3389/ fimmu.2020.01399

Torroba, M., \& Zapata, A. G. (2003). Aging of the vertebrate immune system. Microscopy Research and Technique, 62(6), 477-481. https:// doi.org/10.1002/jemt.10409

Turturro, A., Witt, W. W., Lewis, S., Hass, B. S., Lipman, R. D., \& Hart, R. W. (1999). Growth curves and survival characteristics of the animals used in the Biomarkers of Aging Program. Journals of Gerontology. Series A, Biological Sciences and Medical Sciences, 54(11), 492. https://doi.org/10.1093/gerona/54.11.B492

Witherden, D. A., Verdino, P., Rieder, S. E., Garijo, O., Mills, R. E., Teyton, L., Fischer, W. H., Wilson, I. A., \& Havran, W. L. (2010). The junctional adhesion molecule JAML is a costimulatory receptor for epithelial gammadelta T cell activation. Science, 329(5996), 1205-1210.

Zhou, X., Dou, Q., Fan, G., Zhang, Q., Sanderford, M., Kaya, A., Johnson, J., Karlsson, E. K., Tian, X., Mikhalchenko, A., Kumar, S., Seluanov, A., Zhang, Z. D., Gorbunova, V., Liu, X., \& Gladyshev, V. N. (2020). Beaver and naked mole rat genomes reveal common paths to longevity. Cell Reports, 32(4), 107949.

\section{SUPPORTING INFORMATION}

Additional supporting information may be found in the online version of the article at the publisher's website.

How to cite this article: Emmrich, S., Tolibzoda Zakusilo, F., Trapp, A., Zhou, X., Zhang, Q., Irving, E. M., Drage, M. G., Zhang, Z., Gladyshev, V. N., Seluanov, A., \& Gorbunova, V. (2021). Ectopic cervical thymi and no thymic involution until midlife in naked mole rats. Aging Cell, 20, e13477. https://doi. org/10.1111/acel.13477 